\section{¿Quiénes quieren pedalear? La experiencia de la biciescuela de Ciclofamilia}

Recibido: 20 de febrero de 2018. Aceptado: 9 de abril de 2018.

\begin{abstract}
Resumen
Las políticas y la literatura entorno a la movilidad ciclista presuponen que la conducción de la bicicleta es una actividad sencilla que se aprende en la niñez. Pero ¿es esto cierto? Partiendo de la experiencia de la biciescuela de Ciclofamilia en la Ciudad Autónoma de Buenos Aires y aplicando una metodología Investigación - Acción Participativa, este artículo se propone explorar en las experiencias y motivaciones de las personas que se deciden a aprender a pedalear en la adultez. ¿Es solo una cuestión de biografías personales? ¿Existen sesgos de género entre quienes lograron andar en bici en la niñez y quiénes no? ¿Contemplan las políticas de promoción a quienes no pedalean? El artículo plantea que el aumento de políticas de promoción de la bicicleta genera estímulos positivos para quienes quieren aprender a andar de grande y que estos incentivos impactan de manera diferencial según el género.
\end{abstract}

\section{Who wants to ride? The experience at Ciclofamilia biking school}

Palabras clave

Movilidad Ciclismo urbano Género Autonomía

\begin{abstract}
Policies and literature about cycling mobility assume riding a bicycle is a simple activity learnt during childhood. But is it so? Based on the experience of Ciclofamilia biking school, in Buenos Aires city, and through participatory action research, this article looks into the experiences and motivations of adults learning how to ride a bicycle. Is it just a matter of personal life stories? Is there a gender-based bias between those who learnt how to ride a bike during childhood and those who didn't? Do promotion policies take into account people who don't ride? This work suggests an increase of bicycle promotion policies creates positive incentives for those who want to learn how to ride at an older age, and those incentives have different impacts depending on gender.
\end{abstract}

Keywords

Mobility Urban cycling Gender Autonomy

Palavras-chave

Mobilidade Ciclismo urbano Gênero Autonomia

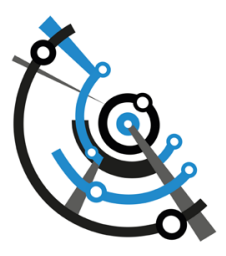




\section{Introducción}

El crecimiento del ciclismo urbano en Latinoamérica durante la última década produjo un efecto sinérgico Estado/usuarios. La existencia de más ciclistas en la calle animó a muchas personas a desarrollar estrategias de movilidad personal basadas en el uso de la bicicleta y a abandonar preconceptos -sobre todo aquellos vinculados a la seguridad vial- en torno al ciclismo urbano.

A su vez, atento a esta demanda creciente y en combinación con los paradigmas de movilidad sustentable, más ciudades de nuestro continente desarrollan políticas cicloinclusivas (BID, 2015) como carriles segregados y una importante oferta de bicicletas públicas. Si bien estas intervenciones se localizan principalmente en las áreas centrales de las grandes urbes, este proceso tiene como efecto principal la ampliación de la legitimidad social de la bicicleta como vehículo en el viario urbano y como solución posible a los problemas cotidianos de movilidad.

El aumento en la cantidad de usuarios y del volumen de las políticas públicas específicas para la bicicleta está teniendo un impacto cada vez mayor en los estudios de movilidad en el ámbito latinoamericano, que se caracterizan por una amplia diversidad de temas y metodologías. ¿Qué intervenciones son necesarias para aumentar la cantidad de ciclistas en la calle? ¿Cuáles son sus impactos, dónde se producen y quiénes resultan beneficiados de las acciones cicloinclusivas? ¿Cuáles son los incentivos para que los conductores dejen de priorizar al automóvil como opción de movilidad personal? ¿Cómo favorecer la integración de la bicicleta al sistema de transporte público?

Un capítulo especial de estas investigaciones relaciona la movilidad ciclista con los estudios de género, especialmente con los usos de la bicicleta que realizan las mujeres en distintos contextos socioterritoriales. Estos trabajos se enfocan en la movilidad cotidiana de las mujeres, en el impacto que las diferencias de género generan sobre los patrones de viaje, los factores que limitan o favorecen en las mujeres la adopción de la bicicleta y si este vehículo puede contribuir a la reducción de las diferencias de género en la movilidad.

Las políticas y la literatura entorno a la movilidad ciclista presuponen cierta universalidad y uniformidad sin distinción de género en las habilidades para la conducción de la bicicleta, ya que es considerada una actividad sencilla que, como la lectura y la escritura, se aprende en la niñez. Pero ¿es esto cierto en todos los casos?

El no haber logrado andar en bicicleta durante la infancia no es tan inhabitual como parece. A medida que se hace más ciclable el ambiente vial y que se instalan algunos consensos respecto de las cualidades ecológicas y saludables del andar en bicicleta en las ciudades, poder pedalear se constituye en una verdadera habilidad social difícil de resolver en la adultez. La tarea de construir espacios de aprendizaje para conducir bicicletas para personas que no lo han logrado en la niñez es una tarea que ha sido abordada casi exclusivamente por los grupos y colectivos de biciactivistas.

A partir de la experiencia de la biciescuela de Ciclofamilia en la Ciudad Autónoma de Buenos Aires, este artículo se propone explorar en las motivaciones y las experiencias de las personas que deciden aprender a andar en bicicleta en la adultez en la hipótesis de que esto aportará a comprender mejor los usos urbanos y expectativas respecto de la bicicleta. ¿Es solo una cuestión de biografías personales o hay algo que podamos indagar para comprender mejor los contextos en que se desarrolla la movilidad ciclista? ¿Existen sesgos de género entre quienes lograron andar en la niñez y quiénes no? 
En una primera sección haremos una revisión muy general de la situación de la movilidad ciclista para entender los contextos que propiciaron la aparición de una biciescuela en la ciudad de Buenos Aires. En una segunda sección haremos una descripción de la metodología utilizada para lograr el pedaleo y de las distintas circunstancias socioterritoriales por las que atravesó la iniciativa. En una tercera presentaremos una sistematización de la experiencia de cinco años de biciescuela, a partir de cuestionarios cerrados y de entrevistas en profundidad para poder conocer quiénes son los "alumnos" de la biciescuela.

Este abordaje lo haremos desde una metodología Investigación-Acción Participativa (IAP) (Ander Egg, 2003). Este encuadre metodológico se presenta como una solución al problema epistemológico de que la autora de este artículo es a la vez la instructora principal de la biciescuela de Ciclofamilia. En el final presentaremos una serie de conclusiones, siempre provisionales, que esperamos resulten en un aporte para la mejor comprensión del fenómeno del ciclismo urbano contemporáneo.

\section{Apuntes sobre la movilidad ciclista en Buenos Aires}

Desde siempre, el centro del mundo de la bicicleta estuvo en Europa, tanto en lo deportivo como en el género de la movilidad cotidiana. Fue el principal vehículo de las clases trabajadoras hasta finales de los años 60 del siglo pasado. Pero, una combinación entre la consolidación del Estado de Bienestar sumada a la imagen de prosperidad asociada al automóvil durante las dos décadas siguientes a la posguerra hizo que muchas familias trabajadoras y de clases medias adoptaran este vehículo como forma de movilidad.

Con la crisis del petróleo surge en los países centrales una creciente preocupación por la dependencia energética y la seguridad vial, principalmente a partir de la denuncia de Ralph Nader sobre el Chevrolet Corvair y el impacto que tuvo en la industria de automóviles a nivel mundial. Para ese momento, en los países nórdicos se desarrollaron durante los años 70 las primeras infraestructuras para la bicicleta, cuando la expansión masiva del automóvil amenazó con colapsar las ciudades.

Algo parecido está sucediendo con la bicicleta en las urbes latinoamericanas a principios del siglo XXI. Como en Europa, luego de que a mediados del siglo XX comenzara un ciclo de fuertes inversiones en infraestructura para el automóvil -ciclo que sigue en nuestros días- las ciudades latinoamericanas redescubrieron las virtudes ambientales y democráticas de la movilidad en bicicleta. Con formas originales, para enfrentar problemas muy diferentes a los que tienen las ciudades modelo europeas, nuestras metrópolis están volviendo a pedalear y con ello a cambiar sus paisajes urbanos.

En todas las grandes ciudades del continente se registran avances muy significativos en infraestructura, en cantidad de viajes y en políticas de promoción de la bicicleta, tal como señala el informe del Banco Interamericano de Desarrollo (BID) titulado Ciclo Inclusión en América Latina y el Caribe (2015), que describe la realidad del ciclismo urbano del continente. El informe, que también ofrece elementos para incentivar el uso de la bicicleta, fue realizado a partir de la consulta a distintas fuentes gubernamentales y no gubernamentales en 56 ciudades de nuestro continente.

El 95\% de las metrópolis latinoamericanas desarrollan en la actualidad políticas de promoción de la bicicleta que van desde la construcción de infraestructuras e instalación de sistemas de bicing hasta iniciativas como bicicleteadas, ciclovías recreativas, semanas de la bicicleta y campañas de seguridad vial. Junto a esto, el hecho de que el $47 \%$ de las ciudades estudiadas tenga un área gubernamental específica dedicada a la movilidad en bicicleta, como en México DF, Montevideo, Medellín, Río de Janeiro y en Argentina; Rosario, Córdoba y Buenos Aires; da un idea de la importancia cada vez 


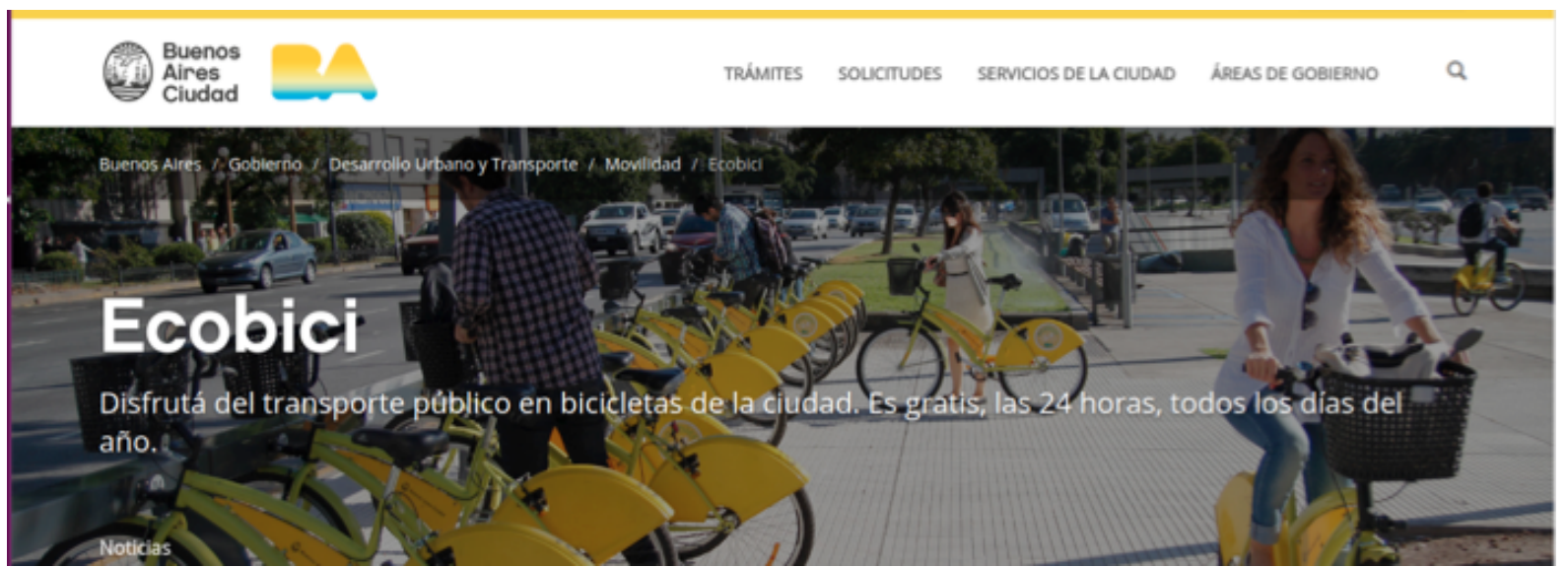

mayor que se le otorga a la bici en las políticas de transporte. Pero el informe advierte también sobre la poca información existente sobre usos de la bicicleta; de hecho sólo el $18 \%$ de la ciudades estudiadas declararon poseer datos que permitan medir el impacto de las políticas ciclistas en el tiempo (BID, 2015).

Pero ¿quiénes son los sujetos de estas iniciativas? Si nos dejamos llevar por las imágenes que promueven el ciclismo urbano en los gobiernos locales (Figura 1) veremos que el usuario objetivo de estas políticas -al menos en su fase publicitaria- son personas jóvenes y adultas de clase media con necesidades de movilidad plausibles de ser satisfechas con la bicicleta. Inclusive se apunta a generar un cambio modal auto-bici en estas poblaciones adjudicando a la bicicleta cualidades similares a las de las publicidades de los automóviles: rapidez, agilidad, libertad y, con el desarrollo en autos híbridos y eléctricos, amigable con el medioambiente.

$\mathrm{Al}$ atender a esta población de ingresos medios y altos, construyendo infraestructura para sus desplazamientos y asignando cierto prestigio social y ciudadano al hecho de pedalear se produce una paradoja: aumentan las opciones de movilidad para quienes ya la tenían resuelta restándole pasajeros al transporte público, aunque de manera marginal.

Si nos enfocamos en el caso de la ciudad de Buenos Aires -que es dónde surgió la experiencia de biciescuela de la que trata el artículo- el crecimiento de la participación modal de la bicicleta pasó < de 0,3 a 3,5 de los viajes totales de acuerdo a declaraciones de la Subsecretaria de Movilidad Sustentable para el período 2007- 2017 (ANSV, 2017:6). Este crecimiento produjo una diversificación modal, siempre deseable en espacios viales caracterizados por la hegemonía del automóvil; pero no produjo ningún impacto sustantivo en la reducción de automóviles particulares circulando por la ciudad.

Siguiendo con el caso de Buenos Aires, la presencia de más bicicletas tampoco implicó reformular los postulados de la ingeniería vial urbana convencional "desde atrás del parabrisas" (Sanz, 2005). La solución encontrada fue la de construir carriles segregados de dos direcciones en calles laterales y -en algunos casos- invadir los espacios de veredas con la consiguiente externalización de los costos de la movilidad ciclista sobre la peatonal.

La opción de instalar carriles segregados en las calles laterales tiene como principal consecuencia un uso diurno en desmedro del nocturno, ya que la luz constituye un factor crítico para la seguridad vial y ciudadana de los ciclistas, especialmente para las mujeres. Por otra parte, la distribución geográfica de la infraestructura ciclista, tanto de los carriles específicos como la disposición de los puestos de bicicletas públicas, no responde a criterios de equidad e integración de los distintos barrios de la ciudad (Ortiz, 2012; Red de Ciclistas Urbanos, 2015).
Figura 1. Sitio web de bicicletas públicas en Buenos Aires. Fuente http://www.buenosaires.gob.ar/ ecobici (Última visita 17/10/2017). 
El énfasis puesto en las infraestructura para el transporte en bici no alcanza a resolver el problema de la convivencia vial y para ello resulta clave la participación de los usuarios en los diseños de las políticas ciclistas (BID, 2015). Esta necesidad no se condice con la falta de dispositivos institucionales - como defensorías, "mesas del ciclista"- que contemplen las demandas específicas y la "ciencia ciudadana" (Tironi Rodó, Valderrama Barragán, 2017) producida por los propios ciclistas porteños. En Buenos Aires si bien sucedieron algunas convocatorias desde el Estado, estas no alcanzaron regularidad ni sistematicidad como para poder ofrecer algún resultado trazable.

Aún con todas estas cuestiones a revisar para la continuidad de las intervenciones cicloinclusivas, la bicicleta es un modo legitimado de movilidad en la ciudad de Buenos Aires. Esto funciona como un incentivo para aprender a pedalear ya que como para la conducción de automóviles y motovehículos, poseer o no esa habilidad impacta en las opciones de movilidad de las personas.

\section{Saberes viales, saberes corporales}

El principal aporte en los estudios sociales del denominado giro a la movilidad -mobility turn- es haber construido un marco que contiene pero a la vez excede los estudios enfocados solo en las demandas de transporte, para abrir preguntas sobre las relaciones sociales que se construyen en los espacios y los tiempos de la movilidad (Sheller y Urry, 2006).

Parte de esta renovada agenda de investigación incluye entre otras cuestiones las relacionadas con la dimensión corporal y sensorial del transportarse, la percepción del tiempo de viaje y la selección de vehículos y rutas como forma de expresar y construir subjetividades (Pérez López, 2017). Así, las formas de socialización en la conducción de vehículos, la tensión entre normas de tránsito y su cumplimiento como las relaciones de poder que se construyen en el escenario vial son objetos también abordables desde las ciencias sociales (Wright, 2010).

Los contextos de aprendizaje de las habilidades necesarias para la conducción de vehículos son muy relevantes desde esta perspectiva. ¿Qué pruebas deben atravesar los aspirantes para obtener una licencia? ¿Cómo son los procedimientos formales e informales en el que se desarrolla el aprendizaje? Si la conducción de vehículos sucede en un escenario marcado por la inequidad vial (Rinaldi, 2014) ¿qué habilidades y discursos son propiciados por el Estado durante el proceso de obtención y renovación de la licencia?

En el caso de la conducción de bicicletas, son las organizaciones y colectivos de ciclistas los que se ocupan de manera casi exclusiva de brindar capacitaciones "por y para ciclistas" apelando a una especificad de la bicicleta que excede el tradicional paradigma punitivo, normativista y autocéntrico imperante en seguridad vial (Sebastián, 2006).

Un ejemplo en Buenos Aires lo constituye el programa En Bici Seguro de la Asociación de Ciclistas Urbanos, que a partir de recursos en la web y en las redes sociales, los ciclistas pueden obtener materiales gráficos, videos y artículos especializados que abarcan desde el uso correcto del casco, las formas de circular por calles y avenidas, la normativa básica sobre la bicicleta, hasta cómo transportar de manera segura a los niños a bordo de la bici. También realizan salidas de capacitación en el tránsito pero, lo hacen para personas que ya conducen sus bicicletas. 


\section{Hacer que el andar suceda. La biciescuela de Ciclofamilia}

Para la mayoría de las personas el momento para aprender a andar en bicicleta es la niñez, quiénes transmiten ese saber tienen alguna relación familiar o afectiva con el educando y es una actividad sesgada a los ámbitos privados. Las políticas de movilidad activa de los gobiernos locales dan por supuestas estas premisas y, a no ser que medien impedimentos como una discapacidad o alguna enfermedad; tienden a considerar la bicicleta como un vehículo universal, de fácil acceso y conducción. Pero, ¿qué pasa con quienes no lograron aprender a andar en bicicleta durante la niñez y desean hacerlo en su adultez? ¿Deben resignarse con un "ya es muy tarde", "me da vergüenza", o "para qué intentarlo”? ¿Hay algún sesgo de género en esto?

Ciclofamilia es una organización con sede en la ciudad de Buenos Aires, Argentina que se dedica a la promoción del ciclismo urbano desde un enfoque de género promoviendo la equidad vial. Hace ocho años se propuso generar acciones, alianzas y campañas con el objetivo de sensibilizar sobre la potencialidad que tiene la bicicleta para satisfacer la mayoría de las demandas de movilidad familiar y de las mujeres, propiciar la autonomía de1@sniñ@s e incluir la perspectiva de género en los diseños de políticas cicloinclusivas para no volver a caer en una ciudad centrada en las demandas individuales de transporte, como la cuestionada movilidad autointensiva.

Entre otras acciones Ciclofamilia lleva adelante desde hace cinco años una Biciescuela para adultos/as; como forma de responder a la importante cantidad de consultas recibidas mediante la web y en otras organizaciones de ciclistas que reclamaban espacios para personas que no lograron aprender andar en bicicleta durante la infancia.

Estas consultas dispararon a su vez preguntas sobre nuestras creencias y prácticas de activismo vial en torno a la bicicleta. Si realmente la bici es un vehículo de emancipación y lucha contra la violencia vial ¿qué hacer con las personas que no aprendieron a pedalear durante la infancia? ¿será que no saber andar puede ser usado como un estigma (Goffman, 1963) por otros ciclistas? O mejor ¿puede alguien decirse biciactivista y no generar un espacio para aquellos que no saben andar y quieren hacerlo?

Otra interrogación que nos producían estos mensajes se relacionaba con la labor de aprender y enseñar. ¿Desde qué lugar producir el andar, desde qué técnica? ¿Desde un yo bicicletista que "se las sabe todas" sobre la bici? Acostumbrados a pensar que todo saber, especialmente los viales, se aprende en la institución que legitima ese saber y no en el saber mismo, nos frenaba el hecho de no tener un manual ni contar con un certificado de instructor.

Una primera pista para resolver estas preguntas provino de un texto de una educadora y militante norteamericana por la igualdad llamada Frances Willard (1839-1898), que en 1895 escribió el libro Una rueda dentro de otra rueda. Cómo aprendí a andar en bicicleta. En él, relata los pasos que tuvo que atravesar para aprender a pedalear con cincuenta y tres años en el final del siglo XIX. Willard sostenía que el sencillo hecho de que las mujeres resultaran tan aptas para pedalear como cualquier varón, era una demostración práctica, clara e indiscutible de la igualdad entre los géneros.

También teníamos presente la experiencia de Macleta, mujeres arriba de la cleta, una ONG chilena que se especializa en género y movilidad. Este colectivo de ciclistas chilenas sostiene desde hace siete años el proyecto Escuela Bicimujer, donde se enseña a las mujeres las habilidades para conducir una bicicleta, las normativas y las prepara para enfrentar las distintas situaciones que presenta el ambiente vial.

Advertimos una posible conexión; casi todos los mensajes que nos habían llegado provenían de mujeres y de hecho han sido mujeres de todas las edades y en una abrumadora 


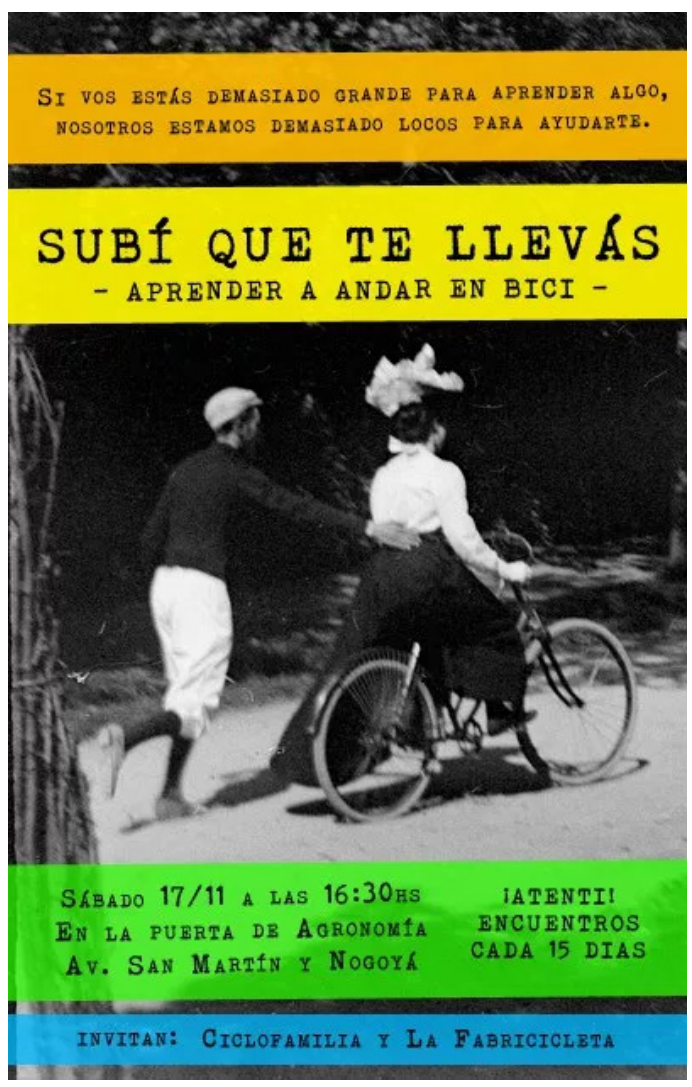

mayoría las que participaron de los encuentros. Pareciera que ellas no sólo están más dispuestas a superar los pudores de no saber andar, sino que como a finales del siglo XIX y a principios del XX, siguen encontrando en la bicicleta un vehículo de libertad y autonomía (ver siguiente sección).

Con todas estas cuestiones en mente, convocamos a un primer taller que ocurrió el 17 de noviembre de 2012. La actividad se produjo en conjunto con el Taller de Ciclomecánica Popular "La Fabricicleta" y la convocatoria fue hecha con un volante digital por redes sociales y en sitios webs con la consigna "si vos estás demasiado grande para aprender algo, nosotros estamos demasiado locos para ayudarte" (Figura 2). La convocatoria tuvo como escenario el parque público de la Facultad de Agronomía de la Universidad de Buenos Aires que dispone de una gran variedad de caminos asfaltados de un ancho similar al de las calles de la ciudad.

Los tres instructores que comenzaron la acción lo hicieron de manera voluntaria, lo que determinó que los talleres tuvieran una frecuencia quincenal; pero para el cuarto encuentro advertimos que esta frecuencia no favorecía la continuidad de la acción. Luego, con la desactivación de La Fabricicleta; Ciclofamilia sostuvo una edición por estación climática de tres encuentros continuados los sábados por la tarde con dos instructores y un piloto de tándem y un máximo de quince participantes de manera libre y gratuita.

Ese formato lo aplicamos para dictar las tres ediciones para los que fuimos convocados por la Secretaría de Extensión Universitaria de la Universidad de San Martín (UNSAM) durante el 2015 y 2016. Actualmente la Biciescuela tiene la forma de una clínica de cuatro encuentros con un instructor y hasta tres aspirantes por turno de dos horas y requiere de un bono contribución para el mantenimiento del parque de bicis y otros gastos asociados a la actividad ya que, con la excepción del período de la UNSAM, la biciescuela no recibió aportes económicos.
Figura 2. Volante de la convocatoria. Fuente: La Fabricicleta y Ciclofamilia. 
La dinámica de aprendizaje que aplicamos para el taller está dividida en tres etapas: ambientación, impulso y consolidación. En la etapa de ambientación, primero se realizan entre dos y tres paseos en tándem de 10 minutos cada uno. A bordo del tándem, el piloto en algún momento levanta las piernas para dejar de pedalear y deja al fogonero con toda la sensación de empujar la bicicleta. Después les participantes se impulsan con los pies en la bici para producir inercia en las ruedas y empezar a aprender a conducir y manipular la bici a velocidad controlada.

En la etapa de impulso, volvemos a hacer lo mismo sobre la bicicleta, pero ahora tratando de poner los pies en los pedales y de pedalear propiamente cuando se va logrando impulso. Este es un momento crítico. El equilibrio no existe como propiedad sino como proceso y está relacionado con el impulso que le imprimamos a la bicicleta desde los pedales. La clave es persistir y estar preparados para tener una nueva sensación de movilidad y hacer que el andar suceda.

Decimos suceda en esta etapa para desmarcarnos de la idea de un sujeto conductor que activa un mecanismo repetitivo (como el encendido del automóvil) y que funciona como una caja negra frente a las órdenes de conducción. En esto, los trenes, los autos y los aviones han ofrecido la idea de un empuje pasivo, donde no tenemos que hacer nada más que operar comandos o pagar un boleto para lograr transportarnos.

La bici nos demanda que nosotros pongamos la energía, que al pedalear sintamos cada círculo de empuje y a la vez podemos ver el mecanismo que produce nuestro desplazamiento. Pero si estamos mirando los pedales y no con la vista al frente, proyectando el camino, seguramente no lograremos que el andar suceda. Desprenderse y a la vez apropiarse de todas las operaciones corporales que son necesarias para conducir una bicicleta es la parte más difícil del aprendizaje.

La consolidación se obtiene cuando los participantes pueden arrancar por sí mismos la bicicleta, mantener un rumbo y doblar con eficacia. En esta etapa se pedalea en parejas o en grupo acompañados siempre por un instructor. Lo que se busca aquí es que los participantes puedan resolver situaciones presentes en la dinámica del tránsito a partir de algunos ejercicios simples como bajar la velocidad de la bici y recuperar el envión sin bajarse de ella, entre otras habilidades. Las clases terminan cuando ya son capaces de conducirse autónomamente en la bicicleta y, a modo de cierre, se les ofrecen nociones de normativa vial y de mecánica.

El método se basa, además de los recursos técnicos ya comentados, en que desconocidos se encuentren con otros desconocidos a resolver algo pendiente. Inclusive, preferimos que personas con un lazo afectivo con el aspirante y que ya saben andar, se abstengan de estar presentes, ya que el pudor es sin duda el principal obstáculo para muchos de los participantes. Este aspecto resulta también una cuestión crítica para lograr pedalear.

\section{¿Quiénes son los que quieren pedalear?}

Sería muy difícil establecer una aproximación al universo total de personas adultas que no saben andar en bicicleta y podrían a adoptarla como modo de transporte. Si bien esto podría resultar relevante de profundizarse las políticas cicloinclusivas, abordar la cuestión desde las metodologías propias de los estudios de movilidad no se justifica en términos de costo-beneficio ya que la incidencia de los que no pedalean tiene, al menos por ahora, un impacto marginal en el desarrollo de las políticas de promoción de la bicicleta.

Pero lo que tampoco se justifica es demorar una acción hasta tanto podamos disponer de un método fiable y resultados metodológicamente consistentes. Entonces ¿cómo aportar elementos para comprender la movilidad ciclista en personas que se declaran 
"inmóviles" respecto de la bici y quieren dejar de serlo? ¿Cómo delimitar un universo legítimo de intervención/investigación desde una plataforma cicloactivista? Una respuesta posible la encontramos en las metodologías propias de la Investigación y Acción Participativa (IAP):

El objetivo del estudio en cuanto a determinar lo que se va a estudiar se decide a partir de lo que interesa a un grupo de personas o a un colectivo. No se trata de estudiar problemas de interés científico o cuestiones que preocupan a un grupo de investigadores, sino los problemas que las personas involucradas consideran importantes porque tienen que ver con cuestiones que conciernen a sus propias vidas. Esto significa, entre otras cosas, que la IAP sólo se aplica a situaciones o problemas de la vida real (Ander Egg, 2003:35).

Esta tradición metodológica se propone comprender a la sociedad al intentar transformarla, transmigrando los objetos de estudio en sujetos de la acción y de la investigación y viceversa. No se trata de acompañar al colectivo de la intervención por razones políticas, ideológicas, o éticas -todas ellas válidas-; se trata de producir una acción con determinados objetivos, medibles y trazables (Ander Egg, 2003). Se trata también de vincular la suerte del colectivo que se está investigando, no solo en la responsabilidad de la tarea compartida sino sobre todo en los resultados de la misma, que constituye un factor crítico para validar esta metodología. En nuestro caso, que los adultos que se contactaron logren andar en bicicleta y construir, a partir de ellos y con ellos; una investigación consistente que sea un aporte para los estudios sobre movilidad ciclista.

Como contamos en la sección anterior, los talleres ocurrieron como respuesta a una demanda de carácter biográfico de muchas personas que se representaron que podría existir un espacio para aprender a andar y nos consultaron como "expertos". Nosotros nos autorizamos a ofrecer el espacio, luego de un proceso no exento de discusiones y mediciones de riesgos.

Si bien contamos con un registro de participantes desde el inicio (Cuadro 1), fue a partir de mayo de 2016, con la dinámica de la escuela ejercitada, que comenzamos a aplicar una metodología IAP para poder contar con una mayor aproximación desde lo cualitativo sobre el universo que nos contactó. Los instrumentos elegidos consistieron en un formulario con preguntas cerradas y abiertas, complementadas por entrevistas a distintas personas que no sabían andar y lo lograron que nos permitiera indagar en las biografías personales para tratar de encontrar elementos comunes que pudieran ser relevantes para la comprensión de la movilidad ciclista. Para eso tomamos el universo de 56 egresados desde mayo de 2016 hasta marzo de 2017. Luego hicimos una devolución de esa investigación con una invitación a escribir un breve relato de no más de 1.000 caracteres que describiera todo lo que implicó el proceso de aprendizaje.

\section{Resultados}

La mayoría de las personas que se contactan preguntando sobre los encuentros desde el primer taller hasta el cierre de este artículo (Setiembre 2017) son mujeres en una proporción del $72 \%$. El $60 \%$ se enteró de los talleres por internet y redes sociales mientras que el 16\% lo hizo por recomendación. La tasa de participación efectiva medida en relación a la cantidad de consultas totales fue de un $45 \%$.

Hicimos un intento de indagar en las razones de la no concurrencia de los que aun consultando y, hasta en algunos casos confirmando su participación, no lo hicieron pero las respuestas que obtuvimos no arrojan resultados concluyentes. Si es lícito inferir por los 
testimonios de los que sí participaron que el hecho de aprender a pedalear de grande resulta muy desafiante, hay personas que lo intentaron en la niñez sin lograrlo y no quieren volverse a encontrar con una frustración. Además los participantes tienen una tendencia a sobrevalorar el riesgo físico al que se van a enfrentar. Estas son, en una primera hipótesis, las principales razones que hacen de la actividad algo plausible de procastinar.

Cuadro 1. Género de las personas que consultan por los talleres en porcentaje. Fuente: Elaboración propia

\begin{tabular}{|c|c|}
\hline Género & Porcentajes \\
\hline Mujeres & $72 \%$ \\
\hline Varones & $28 \%$ \\
\hline
\end{tabular}

En cinco años de biciescuela, lograron egresar 156 personas, de las cuales el 75\% del total son mujeres (Cuadro 2). Esto sugiere un importante sesgo de género ya presente en la cantidad de consultas recibidas.

Cuadro 2. Cantidad total de egresados por género. Fuente: Elaboración propia

\begin{tabular}{|l|c|c|}
\hline & Totales & Porcentaje \\
\hline Egresad@s 2012-2017 & 156 & $100 \%$ \\
\hline Mujeres & 117 & $75 \%$ \\
\hline Varones & 39 & $25 \%$ \\
\hline
\end{tabular}

Lo mismo sucede con las personas que participaron y no lo lograron, en este caso la tasa es del $20 \%$ y se mantiene la proporción de género. La mayoría de este porcentaje no participó de todos los encuentros pactados por cohorte en los que en promedio el andar sucede; por lo que creemos que el no lograrlo está relacionado con no poder o no querer persistir en el intento. Pero no lo sabemos con exactitud y eso es un secreto que pertenece a cada persona.

La franja etaria con mayor participación para el caso de las mujeres se encuentra entre los 30 y 40 años con un 22,6\% y para el caso de los varones está entre los 50 y 60 años con un $28 \%$ sobre el total por género. En ambos sexos, el segundo grupo con mayor número de participantes está entre los 20 y 30 años. Esto podría indicar que debido a que el aumento simbólico ${ }^{1}$ de ciclistas cotidianos se da principalmente en esa franja etaria y en las mujeres, pedalear se estaría convirtiendo en una habilidad importante para la socialización.

\section{Experiencias de los egresados}

La literatura sobre movilidad ciclista otorga un carácter central a las percepciones y sensaciones corporales. Como señala Diz (2016:141) "la bici mantiene al cuerpo en contacto con el entorno, en público y a merced de los elementos, y la acción corporal es condición sine qua non para su funcionamiento". Por lo que nos pareció pertinente indagar sobre esa dimensión a partir de la pregunta “¿Realizas actividad física habitualmente?”, para intentar comprobar sobre si el sedentarismo genera "inmovilidad" ciclista.

El 74\% respondió que practica actividades físicas al menos dos veces por semana en promedio, destacándose el yoga, la natación y el caminar entre las principales. O sea, las personas de nuestro universo que se declararon inmóviles frente a la bicicleta y por eso participaron del curso, no tienen en su mayoría hábitos sedentarios (OMC, 2010) tal como pronosticaba nuestra hipótesis.
1. Decimos simbólico porque esta afirmación no cuenta con respaldo en estudios para el período de referencia, pero como planteamos en la sección anterior, esta es la población objetivo de las políticas de movilidad ciclista del Gobierno de la Ciudad Autónoma de Buenos Aires. 


\section{Edad}

25 respuestas

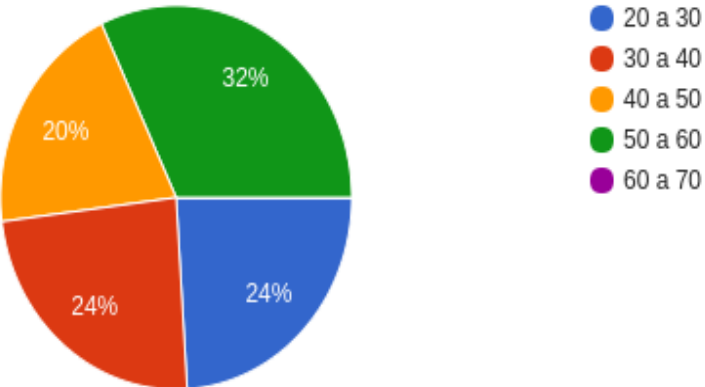

Ya situamos que el momento para aprender a andar en bicicleta es la infancia y que quienes son los encargados de transmitir ese saber poseen alguna relación afectiva con el aprendiz. Pero ¿qué pasa si nada de esto funciona? La mayoría del universo encuestado declaró no contar con experiencias previas con la bicicleta ni en la niñez ni en la adolescencia o la adultez, pero si sitúan algunos intentos siempre discontinuados.

Había perdido las esperanzas de andar en bici, estaba muy bloqueada. Mi novio me quiso enseñar pero yo no me podía ni subir, y aunque él me tenía paciencia yo me sentía presionada y no me salía nada (...) cuando supe del espacio recuperé las esperanzas de poder andar porque me parecía que co-mpartir el aprendizaje con otras personas que estaban en la misma que yo podía estar bueno (C.32 años)

Aquí el sesgo de lo privado parecería ser un factor inhibitorio para lograr que el andar suceda. Pero, como diría el poeta Marechal “de todo laberinto se sale por arriba", en el caso de C., la acción colectiva y cierta institucionalización (Escolar, 2000) del aprendizaje encarnada en la palabra "biciescuela" sirvió como piedra de toque para lograr pedalear.

Podemos encontrar en este testimonio de $\mathrm{H}$. tres aspectos relevantes para comprender más sobre los factores que explican el no aprendizaje en el momento de la niñez.

Nunca pude aprender, mi padre intentó enseñarme pero a mis 10 años desistió.

Vivía en un departamento y lejos de alguna plaza para intentarlo con más frecuencia (H. 26 años)

El primer factor ya fue tratado y es la relación afectiva de quien enseña y la prevalencia del ámbito privado para la transmisión de ese saber. Mientras para aprender a conducir automóviles existen academias, para la bicicleta no. Pero un segundo factor que aparece con nitidez es la poca disponibilidad de espacios adecuados para desarrollar el aprendizaje. En el Área Metropolitana de Buenos Aires la relación metros de espacios verdes por habitante se encuentra en torno a 2,9 $\mathrm{m}^{2} / \mathrm{hab}$. (Observatorio Metropolitano, 2007). Esta situación deficitaria respecto de los espacios verdes ${ }^{2}$ impacta en la pérdida de lugares de recreación públicos y gratuitos para muchos habitantes de la región, especialmente niños, niñas, adolescentes, adultos mayores y personas con discapacidad.

El miedo que genera andar en bicicleta en el ambiente vial o aprender a hacerlo, al igual que otros temores sobre los riesgos que implica la vida en sociedad, tiende a ser producido. En este sentido, la educación vial, las campañas por el uso del casco, "y la
Figura 3. Composición etaria del universo encuestado. Fuente: Elaboración propia.
2. La Organización Mundial de la Salud recomienda que las ciudades deben ofrecer entre 10 a 15 metros cuadrados de espacios verdes por habitante. 
creación de carriles bicis son señaladas como hechos sociales que contribuyen a ver el ciclismo en concreto, y en general la acción de moverse dentro del tráfico, como una actividad peligrosa" (Lorenzi Fernández et al. 2016).

Las ganas de poder andar en bicicleta, dejaron de lado cualquier temor o prejuicio que pudiera tener, era un deseo personal desde hace algunos años que este año me lo propuse como un objetivo. Cuando contás que estás aprendiendo a andar te dicen: ¿te pegaste algún palo? como si fuera una condición inevitable ( 0.29 años)

El temor al riesgo físico es parte también del repertorio de ideas o preconceptos que los participantes tendrían que superar para aprender a pedalear.

$\mathrm{Al}$ ser consultados sobre si tienen pensado incorporar la bicicleta a las opciones diarias de transporte, el 71,4\% contestó que sí lo haría, y al preguntarles si tenían pensado comprar una bicicleta el $74 \%$ respondió afirmativamente. En indagaciones posteriores nos proponemos verificar cuántos de los egresados y egresadas realmente adoptaron la bicicleta como forma de movilidad o si este número representa una "desviación por entusiasmo" por haber logrado pedalear y profundizar sobre aspectos de clase, profesión, etc.

\section{Conclusión}

En este artículo nos propusimos visibilizar a quienes no lograron andar en bicicleta durante la infancia y se propusieron lograrlo a partir del espacio de la biciescuela. El objetivo del trabajo fue obtener elementos relevantes que aporten a los estudios sobre movilidad ciclista en base a las metodologías de Investigación-Acción Participativa. La pregunta central que intentamos responder es si existen elementos que exceden las biografías personales y corresponden al habitus social -en el sentido que le asigna Bordieu- al hecho de no haber logrado pedalear durante la niñez.

Mientras que en un contexto anterior la ausencia de este saber era considerado como algo "pendiente", procastinable y esencialmente privado; el poder pedalear se convirtió en una habilidad social más y a partir del aumento del volumen de las políticas cicloinclusivas en Buenos Aires, difícil de "esconder". Esto sería un incentivo para pasar de lo pendiente a la decisión de aprender y a la búsqueda de dónde. Recordemos que uno de los motivos que originaron la biciescuela fue la cantidad de mensajes consultando sobre un espacio para aprender a pedalear de grande.

Como ya vimos, son principalmente mujeres quienes participan activamente de los talleres y tienen la decisión de aprender a andar en bicicleta en la adultez. Una hipótesis para explicar este sesgo presente en la biciescuela podría ser que las mujeres serían más proclives a asumir que no saben conducir la bicicleta y desearían adoptarla como una opción de transporte más. Ellas verían en la bici una forma de movilidad sin "chaperones" masculinos -como fue el caso de las primeras feministas de finales de XIX y principios del XX- y una forma de ampliar los márgenes de libertad y autonomía.

En las mujeres "se proyecta su rol doméstico sobre el espacio público pero no se disminuyen o eliminan las desigualdades de género. La participación de las mujeres se concentra en cuestiones y tareas vinculadas a las necesidades básicas de la familia y la comunidad. Esta participación es más una obligación por necesidad, que un derecho ciudadano a participar en los asuntos públicos" (Massolo, 2003:32).

La práctica social de moverse es también una relación social que expresa, permite, produce o contesta relaciones de poder. Al tener necesidades específicas y diferentes de 
movilidad en relación a los varones, existen mayores incentivos para que ellas intenten acortar o resolver la brecha de movilidad a las que se ven sometidas utilizando este vehículo (Gutierrez y Leyes, 2017; Linderberg Lemos y otros, 2017).

Siguiendo con esta hipótesis, para el caso de los varones egresados, la bicicleta no sería vista como una herramienta crítica o al menos relevante para solucionar la movilidad cotidiana y la decisión de aprender estaría más relacionada con aprovechar la oportunidad de adquirir un saber más. Esta hipótesis se podría corroborar en la próxima fase del estudio.

¿Por qué hay menos varones entre los participantes? Si bien es esperable que haya más mujeres que hombres que no sepan andar en bici, no creemos que sea en una proporción de 7 a 3. Podríamos decir que el obstáculo estaría en una suerte de estereotipo masculino (Mosse, 2001) relacionado con la conducción de vehículos que hace que tengan una predisposición diferente en relación a lograr o no andar en bici. De cualquier forma, los varones que participaron lograron pedalear en una tasa similar al de las mujeres.

Pero advertimos que, si bien es mucho mayor la participación de mujeres en bicicleta con respecto a la conducción de otros vehículos particulares -19\% para los automóviles, $25 \%$ para la bicicleta (GCBA, 2017)- estos números distan mucho de una situación de paridad. En este sentido, la movilidad no es meramente un reflejo de estructuras sociales, es decir, que solamente las reproduce, sino que también es productora de esas diferencias (Zunino Singh y Jirón, 2017).

Uno de los consensos generales sobre las virtudes de la movilidad ciclista está referido a lo saludable. Como vimos en el universo de los participantes, el hecho de realizar alguna práctica corporal o física sería un incentivo para querer adquirir las habilidades de conducción de la bicicleta, vehículo paradigmático de lo saludable. Por el contrario, los hábitos sedentarios o la ausencia de prácticas corporales podrían explicar la brecha existente entre las personas que consultan sobre los talleres y las que concretan su participación.

La tensión entre la transmisión del saber andar como dominio privado pero que requiere de espacios públicos para desarrollarlo podría ser parte importante de las causas del no aprendizaje en el universo encuestado. Al hecho de que nuestro país tiene el récord de autos por habitante de la región con 3,8 habitantes por vehículo -el $86,7 \%$ son automóviles, el 9,7\% vehículos comerciales livianos y el 3,6\% son ómnibus y camiones (Télam, 2015)- se agrega al déficit de espacios verdes señalado produciendo un ambiente vial objetivamente hostil para ciclistas y peatones (Rinaldi, 2014) y que podría estar relacionado con las discontinuidades en los intentos de aprender que refieren los encuestados. El miedo al riesgo físico y vial con el que cargan la mayoría de los participantes sería una manifestación más específica pero no distinta del miedo social que genera el ambiente vial.

Si bien los ciclistas son parte del "barullo" vial contemporáneo, se mueven en un vehículo que es objetivamente más seguro para los demás y para ellos. Su velocidad es menor, su peso es menor y no tienen parabrisas polarizados que dificultan estructuralmente la visión, entre otras cuestiones. Pensar que un ciclista sin casco es una amenaza para la convivencia vial comparable a la de automovilistas desaprensivos es un tópico recurrente y, a nuestro juicio desproporcionado, en la producción del miedo a la bicicleta en el tránsito.

El objetivo de la biciescuela está ligado a la promoción de la bicicleta como forma de movilidad. La abrumadora mayoría de mujeres egresadas nos reafirmó en la idea de que el uso de la bici puede ser un vector para su inserción como sujetas sociales plenas y promover su derecho a la participación en la esfera pública. Esta es una tarea pendiente de los gobiernos locales y es también un gran desafío para los feminismos latinoamericanos. 
También nos corroboró la propuesta transformadora que implica la adopción de la bicicleta: ser una herramienta bifronte de equidad vial, que acorta la brecha de movilidad entre los géneros y es - en sí misma - herramienta de pacificación vial.

Como dijo Marc Augé en su Elogio, "a la bicicleta le corresponde un papel determinante: ayudar a los seres humanos a recobrar la conciencia de sí mismos y de los lugares que habitan invirtiendo, en lo que le corresponde a cada uno, el movimiento que proyecta a las ciudades fuera de sí mismas para volver a centrarnos en los lugares que vivimos".

Lo más apasionante de la experiencia de la biciescuela es que creíamos que íbamos a enseñar y terminamos aprendiendo. Este trabajo es, a su modo, un agradecimiento a todos los talleristas que se animaron a apostar por su autonomía y en esa labor nos ayudaron a comprender mejor muchas cosas sobre la bicicleta y la movilidad ciclista que ni siquiera habíamos imaginado. 


\section{Q Bibliografía}

» ADEFA (2017). Evolución del Parque Automotor Argentino. Centro Tecnológico de Transporte y Seguridad Vial, Universidad Tecnológica Nacional http://ondat. fra.utn.edu.ar/?p=931, Ultima visita 01/02/2018.

" Agencia Nacional de Seguridad Vial (2017). Voces de la Seguridad Vial. Num. 2. Diciembre 2017. (http://voces.seguridadvial.gob.ar/voces/2017/diciembre/ revista/Revista_voces_ed.2_dic_2017.pdf. Última visita 01/02/2018.

»Ander Egg, E. (2003). Repensando la Investigación Acción Participativa. Colección Política, Servicios y Trabajo Social. Lumen Humanitas.

》Augé, M. (2009). Elogio de la bicicleta. Barcelona: Gedisa Editorial.

" BID (2015). Ciclo-inclusión en América Latina y el Caribe. Guía para impulsar su desarrollo. En internet https://publications.iadb.org/handle/11319/6808 (última visita 01/02/2018).

" Díaz Vázquez, M. S. (2017). La bicicleta en la movilidad cotidiana: experiencias de mujeres que habitan la Ciudad de México. Revista Transporte y Territorio 16, 112-126

»Diz, C. (2016). Objetos Tirados, Objetos Sagrados. Reapropiación tecnológica de la bicicleta. Revista de Antropología Experimental 16, 147-165.

»Escolar, C. (200o). La recuperación del análisis institucional como perspectiva teórica metodológica. En: Topografías de la Investigación, Cora Escolar comp. Buenos Aires: Eudeba, pp. 29-48.

»Goffman, E. (1963). Stigma: Notes on the Management of Spoiled Identity, Prentice-Hall.

" Gorz, A. (1973). La ideología social del automóvil. Le Sauvage, París, setiembre octubre.

» Gray, M., Watson R. (1978). El libro de la bicicleta. Madrid: H. Blume, Ediciones.

» Gutierrez, A., Reyes, M. L. (2017). Mujeres entre la libertad y la obligación. Prácticas de movilidad cotidiana en el Gran Buenos Aires. Revista Transporte y Territorio 16, 147-166.

» Linderberg Lemos, L., Kohler Harkot, M., Santoro, P. F., Ramos, I. B. (2017). Mulheres, por que não pedalam? Por que há menos mulheres do que homens usando bicicleta em São Paulo, Brasil?. Revista Transporte y Territorio 16, 68-92

» López León, L. (2016). Pedalear en la Red. Bicicleta, ciudad y movimiento social. Revista de Antropología Experimental 16, 53-69.

»Lorenzi Fernández, E., ACERO, C. (2016a). Observación de la calidad de las políticas de movilidad ciclistas desde la participación. Un estudio comparado de Madrid y Sevilla. Revista de Antropología Experimental, 16.

»Lorenzi Fernández, E., ORTEGA BOTELLA, D. (2016b). El Reto de la Movilidad Sostenible. Del cuerpo ciclista al espacio urbano y su observación etnográfica. Revista de Antropología Experimental, 16, 1-9.

» Massolo, A. (2003). El espacio local y las mujeres: pobreza participación y empoderamiento. Revista La Aljaba, Universidad Autónoma Metropolitana, México. 
»Mosse, G. (2001). La Imagen del Hombre. La creación de la moderna masculinidad. Madrid. Editorial Talasa.

»Observatorio Metropolitano (2007). Sistema de Áreas Verdes de la Región Metropolitana (SAV). Consejo Profesional de Arquitectura y Urbanismo, Buenos Aires. <http://www.observatorioamba.org/planes-y-proyectos/rmba/planes/ sistema-de-areas-verdes-de-la-region-metropolitana-sav/ficha>. Última visita 01/02/2018.

"Organización Mundial de la Salud (2010). Recomendaciones mundiales sobre actividad física para la salud. Ediciones de la OMS, Suiza.

»Ortiz, F. (2012). El Sistema de Transporte Público en Bicicleta de Buenos Aires. Laboratorio de Políticas Públicas, Buenos Aires. <http://lppargentina.org.ar/ wp-content/uploads/2014/o5/FO.Sistema-pub-de-bicis-en-CABA.pdf>. Última visita 01/02/2018.

»Pérez López, R. (2017). Vínculos entre la bicicleta utilitaria, recreativa y deportiva: análisis del impacto de los programas Ecobici y Muévete en Bici en la Ciudad de México (2006-2012). Revista Transporte y Territorio 16, 220-234

»Pinto, N. (2016). Mujeres y bicicletas. Una expresión de libertad que trasciende fronteras. Mujeres en Bici, PINTO ÁLVARO Nataly (ed.) f. Ecuador, Quito. FESILDIS.

»Red de Ciclistas Urbanos (2015). ¡Atención! ¡Ciclistas activando!: Conclusiones del Segundo Cicloencuentro de la Red de Ciclistas Urbanos. Buenos Aires, 8 de agosto de 2015.

» Rinaldi, F. (2014). Bicicletas y equidad vial. Hacia nuevas formas de entender el tránsito. Revista Transporte y Territorio 11, 135-139.

» Sanz Alduán, A., Pérez Senderos, R., Fernández, T. (1999). La bicicleta en la ciudad. Manual de políticas y diseño para favorecer el uso de la bicicleta en la ciudad. Madrid. Ministerio de Fomento.

"Sanz Alduán, A. (2005). El viaje de las palabras. Revista SAM nํㅜㄹ, Servei de Medi Ambient de la Diputación de Barcelona.

»Sanz Alduán, A. (2009). Viandantes y ciclistas: atravesando la cortina de humo verde. Ingeniería y Territorio. Revista del Colegio de Ingenieros de Caminos y Puertos 86. Cuarta Época.

»Sebastián, N. (2006). Uso y promoción de la bicicleta como medio de transporte en la Ciudad Autónoma de Buenos Aires. La seguridad en el tránsito en la ciudad de Buenos Aires, Defensoría del Pueblo de CABA.

»Sheller, M., URRY, J. (2006). The New Mobilities Paradigm. Environment and Planning, 207-226.

"Subsecretaría de Movilidad Sustentable y Segura G.C.B.A. (2017). Cómo se mueven las mujeres en la Ciudad de Buenos Aires. Presentación en el Encuentro Internacional Movilidad y Género - Construyendo una movilidad inclusiva y segura. Buenos Aires; 9 y 10 de octubre de 2017.

» TELAM - Agencia nacional de noticias (2015). Circulan 12,5 millones de vehículos. Argentina, 23/05/2015. <http://www.telam.com.ar/notas/201705/190013argentina-vehiculos-flota-circulante.html>.

» Tironi Rodó, M., Valderrama Barragán, M. (2017). Transformando la Bicicleta en Laboratorios Móviles. El caso de un self-tracking ciudadano para la ciudad de Santiago. Revista de Antropología Experimental 16. 
»Wright, P. (2010). Imaginarios, símbolos y coreografías viales: una perspectiva Antropológica. II Congreso Iberoamericano de Seguridad Vial, Buenos Aires, 2022 octubre 2010.

»Zunino Singh, D., Paola, J. (2017). Presentación Dossier. Movilidad Urbana y Género: experiencias latinoamericanas. Revista Transporte y Territorio 16, 1-8.

\section{Recursos en Internet}

»Portal de Activvas Asociación Civil Trabajar Contra la Inseguridad Vial y la Violencia con

》 Acciones Sustentables www.activvas.org Última visita 10/11/2017.

»Portal de Ciclofamilia www.ciclofamilia.com.ar Última visita 10/12/2017.

»Blog de La Fabricicleta www.lafabricicleta.blogspot.com Última visita 10/12/2017.

》 Portal En Bici Seguro www.enbiciseguro.org.ar Última visita 10/12/2017.

"Portal de la Federación Europea de Ciclismo www.ecf.com Última visita 10/12/2017.

\section{Marcela Espíndola / ciclofamilia@gmail.com}

Licenciada en Comunicación Social (UCSE). Diplomada en Asesoría Parlamentaria (UTN) y en Género y Comunicación (OEA). En los últimos años ha investigado temas vinculados a género y movilidad urbana. Se especializó en comunicación política e institucional en plataformas multimedia y fue asesora parlamentaria y de prensa de distintos legisladores argentinos. 